\title{
Perspectivas recientes en el estudio de la motivación: la Teoría de la Orientación de Meta ")
}

\author{
Jesús de la Fuente Arias
}

Dpto. Psicología Evolutiva y de la Educación

Universidad de Almería

\section{España}

jfuente@ual.es

\section{Resumen}

Los estudios sobre la motivación académica, desde la perspectiva de la orientación de las metas, han tenido gran protagonismo en los últimos años. Sin embargo, este acercamiento conceptual y empírico no es uniforme, evolucionando desde concepciones normativas hacia otras de carácter multidimensional. El presente trabajo analiza las versiones clásica y reformulada de esta teoría, a través de los presupuestos, las evidencias empíricas, las inconsistencias y las investigaciones desarrolladas por ambas líneas de trabajo. Finalmente, evalúa las implicaciones que ha llevado consigo asumir algunos de sus principios, insuficientemente constrastados, en la práctica educativa.

Palabras clave: Motivación, metas, personalidad, escuela.

(*) In Memoriam de P.R. Pintrich. 


\section{Introducción}

La motivación ha sido una variable con una gran importancia, reflejada en el hecho de que cualquier modelo de aprendizaje conlleva explícita o implícitamente una teoría de la motivación (Maerh y Meyer, 1997; Alonso, 1997; Walberg, 1981).

Desde un enfoque escolar o académico, en la actualidad los modelos motivacionales más recientes consideran la motivación como un constructo hipotético que explica el inicio, dirección, perseverancia de una conducta hacia una determinada meta académica centrado en el aprendizaje, el rendimiento, el yo, la valoración social o la evitación del trabajo (García et al., 1998) Además, en este proceso están implicados cuatro componentes (Pintrich y De Groot, 1990): el valor que los alumnos dan a las metas, la percepción de sus competencias, las atribuciones causales y las reacciones emocionales.

La proliferación de términos diversos y teorías relacionados con el constructo psicológico motivación ha sido históricamente muy amplia. Probablemente la causa haya que buscarla en la dificultad para definir, conceptuar y operacionalizar dicha construcción psicológica con un cierto consenso, existente de forma parcial aún en la actualidad. Los diferentes modelos y teorías sobre la motivación siguen aportando numerosa fundamentación conceptual y evidencia empírica que coexisten en el ámbito de estudio de la motivación: la teoría de la autoeficacia (Bong, 1997; Zimmerman, 2000; Zimmerman y Bandura, 1994; Zimmerman et al., 1992), teoría de la expectativa-valor (Wigfield y Eccles, 2000), teoría de la atribución causal (Miller, Ferguson y Byrne, 2000; Montero y Alonso, 1992; González-Pienda y cols, 2000) o la teoría de la motivación intrínseca-extrínseca (Ryan y Deci, 2000).

Para realizar una categorización de este panorama Murphy y Alexander (2000) han efectuado una revisión de trabajos publicados en los últimos cinco años sobre la terminología utilizada en la investigación sobre las relaciones entre la motivación y el rendimiento académico, identificando un corpus de términos motivacionales que califican como excesivamente variada, con inflación de términos y utilizados de forma difusa. Concluyen que los estudios sobre motivación están centrados en diferentes tendencias: enfoque de metas, motivacional intrínseco-extrínseco, enfoque el interés y las variables de autoesquema. Finalmente, constatan la actualidad, preponderancia e importancia de las aportaciones de la Teoría de Meta en el ámbito de estudio de la motivación. 


\section{La Teoría clásica de la Orientación de Meta: una teoría normativa de las metas}

\section{Fundamentación conceptual}

La visión cognitiva de la motivación ha ido acercándose progresivamente al estudio de las representaciones que hacen los alumnos de las situaciones y, especialmente, las representaciones de las metas como motivos que cada alumno construye (Ames, 1992, Dweck, 1986, Urdan, 1997, Urdan y Maerh, 1995). El papel de las orientaciones de meta es una línea investigadora de gran actualidad, con aportaciones relevantes en el campo de la motivación de logro y el aprendizaje autorregulado (Pintrich 2000a; Pintrich y García, 1994; Pintrich y Schunk, 1996). La importancia y consideración que tiene esta teoría viene reflejada en el hecho de que el Annual Review of Psychology haya publicado recientemente un artículo sobre la misma (Covington, 2000).

"Las metas están referidas a representaciones cognitivas, potencialmente accesible y conscientes... No son rasgos en el sentido de personalidad clásicos, sino representaciones cognitivas que pueden mostrar estabilidad, así como sensibilidad contextual" (Pintrich, 2000a, p. 103). "Representan una unidad de conocimiento estructurada o concepción subjetiva, personal o "teoría"... sobre los propósitos de una tarea de logro, así como a otros elementos referidos a cómo se define el éxito y la competencia, el papel del esfuerzo y errores y normas de evaluación. Estos elementos se activan conjuntamente -el esquema y la teoría- o individualmente, buscando la información pertinente en el contexto... o a través del pensamiento explícito consciente y el conocimiento sobre la tarea de logro" (Pintrich, 2000a, p. 97). Los modelos de representación cognitiva han sugerido que la cognición es más bien un estado, fluctuando entre los factores contextuales inmediatos y las representaciones interiores (Smith, 1998). Desde este modelo las metas pueden conceptuarse como parte de una red de conexiones entre los aspectos diferentes de las metas así como las estrategias y los medios para obtenerlas o, también, como el eslabon cognitivo entre las conductas específicas y los motivos generales (Shan y Kruglanski, 2000), con una cierta estabilidad en los sujetos (Seifert, 1996).

El presupuesto básico de los modelos normativos sostiene que los alumnos pueden ser clasificados según el tipo de meta académica que asuman. Consecuentemente, existirán variaciones en el procesamiento cognitivo y en el proceso de regulación del aprendizaje, siendo los 
alumnos con mayor autorregulación los que manifiestan mayor grado de compromiso con su aprendizaje, los que analizan más las demandas de la escuela y los que más planifican y ejecutan sus recursos y controlan su proceso de aprendizaje (Pintrich, 1999, Zimmerman y Kintasas, 1997). Los modelos normativos de orientación de meta establecen diferentes tipos de metas: las metas académicas y las metas sociales.

Las metas académicas se refieren a los motivos de orden académico que tienen los alumnos para guiar su comportamiento en el aula. Como tales metas pueden promover que los alumnos persigan diferentes objetivos en la situación escolar o académica:

1) Metas de aprendizaje o de dominio (learning, mastery, task or task-involved goal. Denominadas de forma diferente como metas de tarea (Anderman y Midgley, 1997; Kaplan y Midgley, 1997; Middleton y Midgley, 1997) o metas de maestría (Ames, 1992). Este tipo de metas orientan a los alumnos hacia un enfoque de aprendizaje caracterizado por la satisfacción por el dominio y realización de la tarea, con mayores niveles de eficacia, valor de la tarea, interés, afecto positivo, esfuerzo positivo, mayor persistencia, mayor uso de estrategias cognitivas y metacognitivas y buena actuación (Pinchich, 2000b).

2) Metas de rendimiento o de actuación (performance goal). Denominadas como metas de centradas en la capacidad (Nicholls, 1984, Thorkildsen y Nicholls, 1998). Estas metas orientan a los estudiantes a una mayor preocupación por su habilidad, y estar pendientes de la actuación de los otros y parece enfocar a los estudiantes en las metas de hacer las tareas mejor que los demás. En general se ven estas metas como menos adaptativas, por el tipo de motivación asociada a ellas, los efectos emocionales, el menor uso de estrategias y la peor actuación (Ames, 1992, Pintrich, 2000a, Pintrich y Schunk, 1996; Urdan 1997).

3) Metas centradas en el yo (work avoidance, ego or ego-involved goal) (Skaalvik, 1997). Se refieren a las ideas, juicios y percepciones de habilidad desde un punto de referencia normativo y comparativo con respecto a otros. Algunos autores las han clasificado en las dimensiones de acercamiento y defensivas (Elliot y Harackiewicz, 1996; Skaalvick, 1993). 
No obstante el mayor problema a la hora de categorizar conceptualmente los tipos de metas es la diversidad de taxonomías. Anderman y Maerh (1994) han establecido una categorización conceptual de las metas limitándose a las de aprendizaje y de rendimiento. Sin embargo, algunos trabajos más recientes han completado este panorama. Urdan, Anderman, Anderman y Roeser (1998) en un trabajo dirigido a diseñar un instrumento para la evaluación de las metas, han propuesto metas orientadas al aprendizaje, a la aproximación al rendimiento y a la evitación del rendimiento.

Otro supuesto esencial de la teoría reside en la idea de que las metas académicas tienen importancia porque funcionan como un mecanismo de activación para un determinado tipo de procesamiento de la información. Así, las metas de aprendizaje deben llevar consigo un nivel de procesamiento estratégico-profundo, que garantiza el éxito escolar y académico, mientras las metas de rendimiento provoca y un procesamiento de la información repetitivo y superficial, que influye negativamente en el logro final. Por lo tanto se postulan relaciones entre tres elementos diferentes tal y como ha sintetizado Covington (2000): metas <--> cogniciones $<-->$ logro.

Las metas sociales se refieren a las razones de orden prosocial que los alumnos pueden tener para comportarse en la situación académica. Aunque en la actualidad la comprensión del papel que juegan las metas sociales en el aprendizaje es menor que las metas académicas, han comenzado a aparecer resultados que avalan la importancia de este tipo de metas y permiten establecer algunas generalizaciones (Wentzel, 1996; Wentzel y Wigfield, 1998). Algunas investigaciones han encontrado que los alumnos pueden tener metas de orden prosocial, tales como ganar la aceptación de los otros (Schneider, Ackerman y Kanfer, 1996).

\section{Aportaciones empíricas}

\section{Metas académicas, aprendizaje y rendimiento}

\section{Metas académicas y estrategias de aprendizaje.}

Seifert (1995), a través de análisis de cluster, aporta un perfil de grupos de alumnos con diferentes metas (tarea, mejora del yo y evitación del yo) coincidentes con estrategias 
motivacionales diferenciales, en el nivel de autoeficacia, autoestima, emociones, atribuciones y uso de estrategias de aprendizaje, concluyendo la superior puntuación obtenida por los alumnos con perfil de meta de aprendizaje.

En nuestro país, Núñez y otros (1995) han aportado resultados similares con los anteriormente expuestos, aunque reflejan algunas inconsistencias. Las metas de aprendizaje y de reconocimiento social, aparecen asociadas positivamente con respecto a las estrategias de aprendizaje y negativamente con las dificultades en el estudio. En un trabajo posterior del mismo grupo, Roces y otros (1999) han informado de correlaciones positivas aunque moderadas de las metas intrínsecas y extrínsecas con el rendimiento académico.

Middleton y Midgley (1997) han informado, en un estudio correlacional, de relaciones entre las metas de aprendizaje, de rendimiento y de evitación, obteniendo que las metas de aprendizaje correlacionan positivamente con las estrategias de autorregulación y las expectativas de autoeficacia y negativamente con la evitación de búsqueda de ayuda. Las metas de acercamiento al rendimiento correlacionan positivamente las metas de evitación, con la ansiedad de prueba y la evitación de búsqueda de ayuda, y negativamente con el peor rendimiento. Las metas de evitación del rendimiento correlacionan positivamente con la ansiedad de prueba, la búsqueda de ayuda y negativamente con la autorregulación y la autoeficacia.

Skaalvik (1997), en un estudio referido a las metas centradas en el yo, informa de la asociación positiva de las metas de aprendizaje con el autoconcepto académico, autoeficacia y autoestima, mientras la metas de evitación se relacionan negativamente con el autoconcepto académico. Las metas de aumento del yo se relacionan positivamente con el autoconcepto, autoeficacia, trabajo escolar y autoestima, mientras las metas autodefensivas del yo, se relacionan con una alta ansiedad.

\section{Metas académicas y autorregulación en el aprendizaje.}

Las metas de aprendizaje también tienen relación con los procesos de aprendizaje autorregulado. Diversos trabajos han dado cuenta del grado en que las diferentes metas de logro influyen en la regulación del aprendizaje. Winne (1997) ha propuesto un modelo de autorregulación en el que la selección de meta es una estrategia de autorregulación importante a la hora de planificar la acción y el proceso de aprendizaje. También ha aportado evidencia res- 
pecto al papel que la elección de las metas tiene como táctica motivacional, mostrando cómo la orientación de meta depende de la representación de la tarea que realice el alumno a partir de sus ideas y conocimiento de la misma, el dominio del conocimiento, el conocimiento de estrategias de aprendizaje y sus múltiples ideas motivacionales. Zimmerman y Kintasas (1997) han estudiado los efectos de utilizar las metas de proceso y las metas de producto en una tarea autorregulatoria de carácter instrumental, encontrando que los alumnos con metas de proceso tuvieron asociada más autoeficacia, percepción de habilidad y motivación intrínseca; sin embargo, el mejor rendimiento lo obtuvieron los alumnos que en la fase final de la ejecución cambiaron a metas de resultado.

Los estudios correlacionales y de laboratorio han mostrado que los alumnos con metas de aprendizaje se comprometen más en la autorregulación de su aprendizaje (Ames, 1992, Dweck y Legget, 1998; Pintrich y De Groot, 1990), realizan mayor esfuerzo por aprender y tienen un control de la comprensión de lo que se está aprendiendo, dándose cuenta de lo que están aprendiendo y lo que no (Middleton y Midgley, 1997), utilizan más estrategias de parafraseo y resumen para comprender (Archer, 1994; Archer y Scevak, 1998), realizan atribuciones más adaptables para comprender sus fracasos, tienen más sentimientos de orgullo y satisfacción en el éxito y menos ansiedad ante el fracaso (Ames, 1992). Los resultados no son tan concluyentes para los alumnos que asumen metas de rendimiento, aunque existen estudios que han mostrado que este tipo de metas llevaba consigo estrategias superficiales y repetitivas (Karabenick y Collins-Eaglin, 1997). En el caso de los alumnos con metas de evitación de fracaso, los resultados muestran un modelo de tenacidad reducida para el esfuerzo (Bouffard, Vezeau y Bordeleau, 1998).

También se han relacionado las metas de aprendizaje con las características en dificultades de aprendizaje de los alumnos. En un estudio reciente González-Pienda y cols. (2000) han comparado las metas entre los alumnos y las características en dificultades de aprendizaje de los mismos, informando de diferencias significativas entre los alumnos con y sin dificultades de aprendizaje (DA). Los alumnos sin DA tienen de forma significativa más de aprendizaje y relacionadas con el yo, mientras no aparecen diferencias significativas entre ambos en cuanto a la metas de rendimiento. 


\section{Metas académicas y estrategias de autorregulación de la motivación.}

Aún existe una cantidad limitada de investigación educativa que delimite cómo los estudiantes regulan su nivel de motivación y si las estrategias que les permiten mantener o aumentar su esfuerzo para terminar las tareas, son un componente importante del aprendizaje autorregulado. Diferentes trabajos han mostrado cómo los alumnos se implican por controlar su esfuerzo continuado. El modelo de autorregulación del esfuerzo (Kulh, 1984, 1985, 1992) ha revelado que los estudiantes trabajan para conseguir una determinada meta, tras ser elegida, a través de una variedad de estrategias de control voluntario. Zimmerman y Martínez-Pons $(1986,1990)$ han proporcionado evidencia en cuanto al uso de estrategias para mantener la persistencia en las tareas académicas ante las alternativas distractoras e interesantes.

Las metas académicas se han mostrado como una importante variable que puede ayudar a delimitar este problema, al ser incorporadas a los modelos de autorregulación de la motivación. Volet (1997) ha utilizado dos dimensiones de las metas académicas (dirección y esfuerzo), mostrando la necesidad de que se produzcan conjuntamente para obtener un buen rendimiento académico. El esfuerzo aparece como una estrategias de regulación motivacional de primer orden, predictora del rendimiento académico (Boekaerts, 1994). Wolters (1998) ha mostrado cómo los alumnos ajustan las estrategias de autorregulación motivacional para conseguir una meta previamente elegida, en diferentes situaciones simuladas. Los alumnos mantienen su compromiso motivador de forma activa de la misma forma que utilizan estrategias de regulación cognitiva. Los alumnos con metas orientadas al aprendizaje utilizan estrategias motivacionales más intrínecas, mientras los de metas orientadas al rendimiento ponen en práctica estrategias motivacionales más extrínsecas.

\section{Metas académicas y cambio conceptual.}

En los modelos actuales sobre cambio conceptual se considera importante tomar en consideración las ideas motivacionales de los alumnos como las estrategias metacognitivas a la hora de explicar cómo se produce el cambio conceptual en los alumnos. Pintrich (1999) ha propuesto diversas concepciones motivacionales de los alumnos que mejor facilitan el cambio conceptual. Entre otras concepciones, la adopción, por parte del alumno, de metas de aprendizaje y conocimiento probabiliza el cambio conceptual. Diversos estudios fundamentan esta propuesta, al mostrar la asociación encontrada en alumnos con metas orientadas al aprendizaje 
y al conocimiento, con respecto a las estrategias de procesamiento, con un mayor nivel de elaboración de la información, estrategias de autorregulación y de reflexión (Pintrich, 2000a; Pintrich y De Groot, 1990, Pintrich y García, 1994).

También los modelos de cambio conceptual de los profesores han incorporado aportaciones de la teoría de meta. Patrick y Pintrich ( 2001) han propuesto que las concepciones de los profesores sobre el aprendizaje llevan consigo orientaciones de meta implícitas, así como diseños de enseñanza que favorecen determinados tipos de orientaciones en los alumnos.

\section{Metas académicas, aprendizaje y rendimiento}

Las relaciones directas entre metas y resultados académicos han aparecido en estudios causales (Roney, Higgins y Shah, 1995; Roney y Sorrentino, 1995; Schunk, 1996). También han sido demostradas en numerosos estudios las relaciones entre la calidad de procesamiento cognitivo y los resultados académicos, mostrando que el nivel de procesamiento profundo está asociado al rendimiento (Covington, 1992).

Diversos estudios de regresión múltiple han confirmado la asociación entre las metas de rendimiento o evitación de fracaso con procesamiento superficial y la desorganización de la planificación del estudio, factores asociados a la vez a un peor rendimiento académico, mientras que las metas de aprendizaje aparecieron asociadas al procesamiento profundo, tenacidad, alto esfuerzo y, finalmente, al logro elevado (Elliot McGregor y Gable, 1999).

\section{Metas académicas y determinantes personales}

\section{Concepciones de los alumnos y metas académicas.}

De entre los diferentes factores que pueden influir en la construcción de determinadas metas por parte de los alumnos una de las que ha aparecido con mayor peso una de ellas es la concepción que tienen los alumnos sobre la inteligencia. Según Nicholls (1984) y Dweck (1986), las concepciones de los sujetos sobre la inteligencia están asociadas a las metas asumidas en las situaciones de aprendizaje. Los alumnos que entiendan la inteligencia como algo fijo, estable y diferenciado del esfuerzo (rasgo estable) asumirán más probablemente metas de 
rendimiento, mientras que los que conciban aquella como un rasgo cambiable y modificable en función del esfuerzo (rasgo incremental), asumirán metas de aprendizaje.

Uno de los indicadores de esta concepción son las atribuciones realizadas por los alumnos ante las situaciones de éxito y fracaso en las tareas académicas. Se ha encontrado que los alumnos con un estilo atribucional internalista construyen metas de aprendizaje, mientras los alumnos con atribuciones más externalistas asumir metas de rendimiento (Valle, González, Gómez, Rodríguez y Piñeiro, 1998).

\section{Personalidad y metas académicas.}

Algunos autores han reconocido la necesidad de incorporar diversas variables de personalidad, insuficientemente representadas en la actualidad, en los modelos explicativos de la motivación de logro (Covington, 2000). Pintrich (1999) ha planteado la posibilidad de entender las metas como una variable estable y de orden disposicional, más bien que como una simple respuesta a las demandas situaciones. Otros autores han sugerido que las metas académicas están moduladas por variables contextuales y de personalidad, de forma interactiva, proponiendo que las metas pueden tener un efecto motivador diferente en los alumnos, dependiendo de las variables de personalidad, existiendo alumnos que desean implicarse en tareas competitivas mientras otros la evitan (Harackiewicz et al., 1997; Harackiewicz, Barron, Tauer, Carter y Elliot, 2000).

La teoría de la autovaloración (Covington, 1992, 1998, 2000) ha postulado la importancia y necesidad que tienen los alumnos de mantener su valía personal. Igualmente ha tomado en consideración diversas estrategias protectoras que utilizan los alumnos para el mantenimiento de la misma. De acuerdo con ésta teoría, Thompson (1994) establece tres tipos de estrategias autoprotectoras:

1. estrategias de protección de la autovalía (self-worth protections): consiste en no realizar esfuerzo cuando se anticipa el fracaso (Thomson, Davison y Barber, 1995; Covington, 1998).

2. estrategias de auto-impedimento (self-handicapping): creando tácticamente alguna causa (real o figurada) que impida la realización de la tarea y estableciendo metas poco realis- 
tas de logro (Covington, 1992; Martin, Marsh y Debus, 2001). Migdley y Urdan (1995) han encontrado que los alumnos con bajo rendimiento utilizan más estrategias de autoimpedimento que los de alto rendimiento, para dejar a salvo su falta de habilidad.

3. estrategias de pesimismo defensivo (defensive pessimism): manteniendo expectativas excesivamente bajas para minimizar el esfuerzo, garantizar el éxito y minimizar la ansiedad que produce la no consecución del éxito, con consecuencias como el agotamiento o el bournout (Urdan et al., 1998)

\section{Género y metas académicas}

En términos generales, los resultados avalan la idea de que las metas de aprendizaje y sociales están asociadas en mayor cuantía al género femenino, mientras que las metas de rendimiento lo están al género masculino (Wentzel, 1998). Thorkildsen y Nicholls (1998) han informado de una mayores metas de aprendizaje en las alumnas y de mayores metas centradas en el yo, de rendimiento y de evitación en los alumnos. Igualmente las alumnas aparecen con mayores atribuciones de interés y esfuerzo, mientras los alumnos dan explicaciones más extrínsecas de los sucesos relacionados con el rendimiento.

\section{Características de riesgo social, metas académicas y sociales}

Carroll, Baglioni, Houghton y Bramston (1999) han mostrado la existencia de diferencias significativas entre alumnos de alto y bajo riesgo social en cuanto a sus metas académicas. Mientras los primeros parecen lograr una buena autoimagen académica, ligada a las superiores metas educativas e interpersonales, los segundos aparecen buscar una buen imagen social, con metas sociales y físicas superiores, destacando la búsqueda de reputación social.

\section{Metas académicas y Contexto de aprendizaje}

\section{Metas del contexto y estrategias autoevaluativas}

La dinámica del aula puede moderar los efectos de meta en los alumnos. Las aulas con metas competitivas de capacidad o de evitación del fracaso favorece que los alumnos dejan de prestar atención y valorar la importancia del aprendizaje o desempeño para centrar su 
atención o esfuerzo en superar a los otros, mientras las aulas con metas de aprendizaje promueven numerosas gratificaciones, involucrando a los alumnos en un compromiso con su aprendizaje, reconoce el esfuerzo de los alumnos, promueve el aprendizaje a partir de los errores o explicitando las metas (Harackiewicz y Barron, 1998; Harackiewicz, Barron, Carter, Letho y Elliot, 1997). Slavin (1983) estableció algunos elementos definitorios de la situaciones de aprendizaje promotoras de metas de aprendizaje: la posibilidad de elección de tarea, la elección de metas individuales y la autonomía en la acción escolar.

Diversos trabajos han analizado el papel de la estructura de meta de clase en el uso de las estrategias de auto-dificultad en los alumnos (Maehr y Midgley, 1996; Midgley, 1993; Migdley, Arunkumar y Urdan, 1996; Urdan, Midgley y Anderman, 1998). Las metas personales de evitación del rendimiento y la percepción del aula como una situación con metas de rendimiento predicen la utilización de las estrategias de auto-dificultad. Las metas de aprendizaje y de rendimiento no predicen su uso (Midgley y Urdan, 2001). La percepción de los alumnos de la estructura de meta del aula ha sido considerada como más decisiva que la propia estructura del aula (Ames y Archer, 1988; Maehr y Midgley, 1991). Los resultados de este estudio revelan que la utilización de estrategias de auto-dificultad están asociadas positivamente con la percepción de meta del aula centrada en el rendimiento, con una peor percepción de competencia, peor nota media y con el sexo masculino. Estos resultados son consistentes con la evidencia previa que muestra la mayor probabilidad en cuanto a la aparición de este tipo de estrategias en aulas con metas de rendimiento para proteger su autovaloración (Covington, 1992; Pajares y Kranzler, 1995; Ryan y Pintrich, 1997).

\section{Metas de contexto, estrategias de aprendizaje y rendimiento.}

Algunos trabajos han intentado mostrar la relación entre las metas académicas propuestas en la situación de aprendizaje y el uso de estrategias de aprendizaje. Karabenick y Collins-Eaglin (1997) han mostrado que las aulas que utilizan incentivos de trabajo en grupo correlacionan positivamente con el uso de estrategias de elaboración, pensamiento crítico y metacognición. Además, las metas de rendimiento han apareciendo negativamente asociadas al uso de estrategias de elaboración y de pensamiento crítico. Schunk (1996), utilizando contextos de aprendizaje y de rendimiento, con autoevaluación implicativa y no implicativa, han mostrado los efectos de estas situaciones en las expectativas de autoeficacia de los alumnos, la persistencia en el esfuerzo y las tendencias de la autoevaluación. 
Bergin (1994), en un trabajo con diseño experimental interactivo, ha puesto de manifiesto los efectos perniciosos que tienen las situaciones de meta competitiva para los alumnos de bajo rendimiento, tanto en una tarea de recuerdo libre como en puntuaciones de examen.

\section{Metas sociales y rendimiento académico}

La meta de búsqueda de amigos es una meta que se da en los niños de todas las edades, frecuentemente con mayor énfasis que las metas académicas (Wentzel, 1991, 1992). Los escolares con mayores metas prosociales son los juzgados más positivamente por sus compañeros y profesores (Wentzel, 1996). El comportamiento social cooperativo, dócil y dispuesto a compartir se asocia positivamente con el rendimiento académico (Wentzel, 1991, 1993). Las metas sociales se asocian con las metas académicas para predecir el logro académico (Wentzel, 1998).

\section{La Teoría Reformulada de la Orientación de la Meta: una teoría de las metas múltiples}

\section{Fundamentación conceptual}

Esta versión de la teoría de meta asume algunos planteamientos nuevos, avalados por la evidencia empírica (Harackiewicz, Barron y Elliot, 1998; Pintrich, 2000b):

1. Las metas de desempeño no tienen que ser necesariamente desadaptativas. Pueden estar asociadas a un buen rendimiento si se presentan unidas a metas de aprendizaje (Elliot, 1997; Elliot y Church, 1997; Elliot y Harackiewicz, 1996; Harackiewicz, et al., 1997; Harackiewicz, Barron y Elliot, 1998).

2. Las metas que asuman los alumnos pueden ser múltiples y flexibles en las situaciones reales de aula, a diferencia de los modelos únicos, utilizados de forma generalizada en los estudios experimentales (Hidi y Harackiewich, 2000). En algunos estudios de aula han aparecido relacionadas positivamente las metas de aprendizaje y rendimiento (Pintrich, 2000a). Por tanto, es posible que la utilización combinada e interactiva de los dos tipos de metas tenga un efecto multiplicativo positivo en el rendimiento, siendo el nivel conjunto y alto en las metas de aprendizaje y de rendimiento lo más adaptativo para los alumnos. 
Además cabe la posibilidad que el nivel de metas de aprendizaje asumido pueda tener cierta dependencia de las metas de rendimiento (Harackiewicz, Barron y Elliot, 1998).

Pintrich (2000b) ha sintetizado la posibilidad de que los alumnos adopten metas diferentes a través de diferentes momentos, obteniendo un buen logro, a través de la "metáfora del viaje":

1) los alumnos con metas de aprendizaje pueden utilizar en el tiempo diversas estrategias de motivacionales, afectivas y de aprendizaje que, al reportarles buenos logros, finalmente, les hagan asumir metas de rendimiento.

2) los alumnos con metas de rendimiento pueden conseguir un buen rendimiento si además de estas metas asumen metas de aprendizaje. Por tanto, más importante que el tipo de meta asumida es si la meta promueve la implicación afectiva y cognitiva en la actividad (Harackiewicz, Barron y Elliot, 1998).

Pintrich (2000a) ha reconocido la importancia de las aportaciones de otros modelos recientes al mostrar la existencia de dos estados -acercamiento vs. evitación- a través de los cuales pueden actualizarse en los sujetos, tanto las metas de aprendizaje como las de rendimiento, sugiriendo que no es correcto definir a las metas rendimiento siempre de forma negativa en contraposición a las de aprendizaje (Hidi y Harackiewich, 2000; Elliot, 1997; Elliot y Church, 1997; Elliot y Harackiewicz, 1996). Estos autores han encontrado que las metas de rendimiento de acercamiento pueden llevar consigo un buen rendimiento, y sólo las metas de rendimiento de evitación están asociadas con pobres resultados.

3. La importancia de las metas sociales. Una línea de trabajo reciente, tradicionalmente menos desarrollada, ha recordado la necesidad e importancia de las metas sociales en el aprendizaje y en el rendimiento académico. Los modelos actuales (Wentzel, 1998, 1999, 2000) postulan la importancia y el posible papel complementario de las metas sociales con respecto a las de aprendizaje y de rendimiento. Este tipo de metas se han conceptuado como representaciones cognitivas referidas con la consecución de objetivos sociales (establecer y mantener relaciones sociales) y están relacionadas con los valores de logro que tiene el sujeto. Los valores proporcionan las razones específicas a los individuos para la persecución de una determinada meta. Wentzel (1999) ha establecido una taxonomía de este tipo de metas: 
1. Metas de relaciones sociales auto-asertivas. Referidas a la consecución de la individualidad, autodeterminación, superioridad y adquisición de recursos sociales.

2. Metas de relaciones integradoras. Referidas a la consecución de relaciones comunes, responsabilidad y compromisos sociales, la equidad o justicia, la provisión de recursos sociales.

Este modelo postula la existencia de relación entre las metas de aprendizaje y las metas sociales a la hora de explicar y predecir el aprendizaje y el rendimiento, postulándose tres modelos diferentes: complementariedad, unidireccionalidad e interdependencia jerárquirca (Wentzel, 2000).

4. Las concepción de un mayor énfasis en el estudio de la interacción entre el individuo y el contexto a la hora explicar las metas múltiples de los alumnos (Wentzel, 1991,1992, 1993, 1996).

\section{Aportaciones empíricas}

\section{Metas académicas, aprendizaje y rendimiento.}

Harackiewicz y cols. (1997) han encontrado correlaciones positivas entre las metas de aprendizaje y las de rendimiento. Bouffard, Vezeau y Bordeleau (1998), desde un enfoque de estudio del cambio que se pueden producir en el tipo de metas en el tiempo, han encontrado que los alumnos de los últimos cursos de la educación secundaria tienden a utilizar metas de rendimiento, a diferencia de los primeros cursos, que lo hacen hacia metas de aprendizaje. Estos resultados se pueden considerar como una estrategia adaptativa de los alumnos a las demandas y requerimientos del propio sistema educativo, lo que da cuenta de la flexibilidad de las metas académicas y del papel de la influencia externa sobre ellas. También aparece asociada la mejor autorregulación a las metas de aprendizaje, en todos los niveles analizados.

Pintrich (2000b) ha obtenido resultados que confirman tanto las hipótesis de trabajo de la teoría de meta reformulada como los de la teoría normativa de meta. A favor de la teoría normativa de meta está el hecho de que la meta de aprendizaje lleva consigo el mejor comportamiento y resultado. A favor de la teoría reformulada, ha aparecido que los comportamientos 
motivacionales de los alumnos, tal y como muestran otros estudios longitudinales, no son estáticos, sino adaptables a los distintos momentos de las tareas y adaptando las metas al momento (Eccles, J., Wigfield, A. y Schiefele, 1998; Wigfield et al, 1996). Por otra, los resultados de su estudio muestran la inexistencia de diferencias entre los alumnos altos en metas de aprendizaje y bajos de rendimiento, con respecto a los alumnos y los altos en metas de aprendizaje y de rendimiento (en la autoeficacia, las estrategias cognitivas y metacognitivas), con algunos resultados a favor de éstos últimos (valor de la tarea).

Wentzel y cols.(Wentzel, 1994; Wentzel y Asher, 1995; Wentzel y Caldwell, 1997) han mostrado en diferentes estudios la relación positiva existente entre las metas de aprendizaje y las metas sociales, apuntando en la dirección de la importancia que tienen las relaciones con los iguales y el tipo contexto para promover metas de aprendizaje. Wentzel (1998) ha mostrado las relaciones entre las distintas metas de aprendizaje. Las metas de aprendizaje correlacionan positivamente con la cohesión familiar, la percepción de apoyo del profesor el interés con lo relacionado con la escuela, con las metas prosociales, las metas de responsabilidad social y el interés en la clase; las metas de rendimiento correlaciona negativamente con la cohesión familiar y con el interés por la escuela; las metas prosociales correlacionan positivamente con la percepción de apoyo del profesor y de los iguales, el interés por lo relacionado con la escuela, las metas de aprendizaje, la responsabilidad social y el interés en clase.

\section{Metas académicas y estrategias de autorregulación de la motivación.}

Zimmerman y cols (Zimmerman, 1994; Zimmerman y Risemberg, 1997) han reflejado las dimensiones conceptuales de la autorregulación académica. En su modelo plantea como primera dimensión conceptual el por qué del aprendizaje, que da lugar a la dimensión psicológica denominada motivación, estableciendo como las variables del proceso de autorregulación en el aprendizaje las metas, las expectativas de autoeficacia, los valores o las atribuciones.

Wolters (1998) ha mostrado que las metas de rendimiento y de aprendizaje son una variable que define las estrategias de regulación motivacional de los alumnos, conjuntamente con otras como la búsqueda de premios extrínsecos, el valor de la tarea, el interés, la eficacia, la búsqueda de ayuda o la fuerza de voluntad. Los alumnos que eligen una determinada meta tienen a esforzarse por mantenerla. En segundo lugar, ha verificado la utilización de diferentes 
estrategias de regulación motivacional ante situaciones problemáticas con diferente grado de dificultad. Ello supone que los alumnos adaptan o modifican el uso de estrategias motivacionales para adaptarse a las demandas circunstanciales. También ha constatado la asociación de orden positivo existente entre las metas de aprendizaje, como estrategias de autorregulación motivacional, y las estrategias cognitivas de elaboración, pensamiento crítico y metacognición, y viceversa con las metas de rendimiento. Sin embargo, no se ha podido comprobar la asociación entre la utilización de meta de aprendizaje, como estrategia regulatoria para mantener la motivación y el rendimiento final. Este resultado sugiere que las metas de aprendizaje cumplen una función de mantenimiento de la regulación en el buen aprendizaje, pero probablemente sea necesaria la utilización de metas de rendimiento, como estrategia regulatoria de la motivación, para obtener un resultado académico óptimo.

\section{Conclusiones, inconsistencias e implicaciones educativas}

La teoría de meta ha supuesto un potente revulsivo en el ámbito del estudio de la motivación de logro académico, al incorporar una nueva variable, esencial para poder conocer y explicar los procesos psicológicos motivacionales que operan en el proceso de enseñanzaaprendizaje. Conjuntamente con otras teorías y modelos más clásicos de la motivación configuran un panorama alentador. No obstante, siguen existiendo inconsistencias en esta teoría, dignas de ser tomadas en consideración para un futuro próximo:

1. El estudio de las metas de los alumnos ha estado sesgado hacia el estudio de las de orden académico, en detrimento de las de carácter social, aunque éstas se estén mostrando con una gran importancia, especialmente en alumnos de contextos educativos más desfavorecidos.

2. El partir de la construcción del binomio antagónico metas de aprendizaje vs. metas de rendimiento ha llevado a la presuposición implítica de una asociación lineal con el buen y mal rendimiento, respectivamente. Tal hipótesis, a pesar de los resultados que la avalan en los numerosos estudios que analizan las metas de los alumnos de forma excluyente, se ha mostrado inconsistente en los trabajos que efectúan el análisis de las metas de forma conjunta, al aparecer resultados de alumnos con un buen rendimiento y regulación del aprendizaje que combinan ambos tipos de metas. 
3. La conceptuación reciente de las metas como un fenómeno multidimensional ha llevado a plantear la conveniencia de reenfocar este campo, desde la visión predominante individual de esta variable hacia una visión interaccionista, en la que confluya el estudio de las metas como variable individual, como variable influída contextualmente y como variable interactiva de factores personales (estables y actualizables) y contextuales. Este enfoque de estudio es el asumido en la actualidad en otros fenómenos del aprendizaje y del rendimiento (Renzulli y Yun, 2001).

Por tanto, cualquier intervención educativa, dirigida a mejorar la motivación de los alumnos, debería asumir un enfoque multidimensional:

1. Incluir las metas de forma integrada con el resto de las variable motivacionales y las estrategias de aprendizaje, enseñando a los alumnos a autorregularlas de forma conjunta (Dembo, 2000).

2. Establecer medidas para la mejora de los procesos motivacionales y de aprendizaje, tanto en un nivel contextual del aula como en los alumnos.

3. Evaluar los posibles efectos interactivos entre los diferentes tipos de metas propuestas a los alumnos a través del proceso de enseñanza, las metas que los alumnos construyen en la situación, dependiendo de sus variables personales y la interacción entre ambas para explicar el aprendizaje y el rendimiento.

Hidi y Harackiewich (2000) se han referido, con bastante precisión, a esta problemática:

"Durante las dos últimas décadas ha existido una fuerte preocupación por reducir las influencias motivadoras externas e intentar dar energía a las fuentes intrínsecas. Este último tipo de meta es muy digno, pero dar protagonismo a las fuentes de motivación intrínseca no significa necesariamente que todas las fuentes extrínsecas sean sospechosas. Evaluar de forma negativa el uso de los motivadores extrínsecos (premios, refuerzos, etc.) y de las metas de logro ha sido el resultado natural de esa visión.

Ha llegado el momento de reevaluar esta situación. La preocupación original sobre los efectos de las influencias externas era una reacción al conductismo. Una consecuencia de 
esta negación es que hemos terminando negando la importancia de las influencias externas que incluyen las motivaciones que puede ser necesario dar a todos los estudiantes para que tengan un decente, no igual, cambio hacia el logro. Además, estamos considerando a los estudiantes que desean destacar e intentan estar entre los mejores como un comportamiento desadaptativo o como una meta políticamente incorrecta. ¿No es esto absurdo? (pág. 169) “.

\section{Referencias}

Alonso Tapia, J. (1997). Motivar para el aprendizaje. Teoría y estrategias. Barcelona: Edebé. Ames, C. (1992). Classrooms: Goals, Structures, and Student Motivation. Journal of Educational Psychology, 84 (3), 261-271.

Ames, C. y Archer, J. (1988). Achievement goals in the classroom: Students' learning strategies and motivation process. Journal of Educational Psychology, 80, 260-267.

Anderman, E.M. y Maerh, M.L.(1994). Motivation and strategy use in science: Individual differences and class effects. Journal of Research in Science Teaching, 31, 811-831.

Anderman, E.M. y Midgley, C. (1997). Changes in achievement goal orientations, perceived academic competence, and grades across the transition to middle-level schools. Contemporary Educational Psychology, 22 (3), 269-298.

Archer, J. (1994). Achievement goals as a measure of motivation in university. Contemporary Educational Psychology, 19, 430-446.

Archer, J. y Scevak, J.J. (1998). Enhancing students' motivation to learn: Achievement goals in university classroom. Educational Psychology, 18 (2), 205-223.

Bergin, D.A. (1994). Efects of a mastery versus competitive motivation situation on learning. Journal of Experimental Education, 63 (4), 303-314.

Bouffard, T., Vezeau, C. y Bordeleau, L. (1998). A developmental study of the relation between combined learning and performance goals and students' self-regulated learning. British Journal of Educational Psychology, 68, 309-319.

Bong, M. (1997). Generality Academic Self-Efficacy Judgements: Evidence of Hierarchical relations. Journal of Educational Psychology, 89 (4), 696-709.

Boekaerts, M. (1994). Action control: How relevant is it for classroom learning? En J.Kuhl y Beckmann (Eds.), Volition on Personality: Action versus state orientation (pp. 427435). Bern: Hogrefe and Huber Publishers. 
Carroll, A., Baglioni J.R., Houghton, S. y Bramston, P. (1999). At-risk and not at-risk primary school children: An examination of goal orientations and social reputations. British Journal of Educational Psychology, 69, 377-392.

Covington, M.V. (1992). Making the grade: A self-worth perspective on motivation and school reform. Cambridge, England: Cambridge University Press.

Covington, M.V. (1998). The will to learn. New York: Cambridge University Press.

Covington, M.V. (2000). Goal Theory, Motivation, and School Achievement: An Integrative Review. Annual Review of Psychology, 51, 171-200.

Dembo, M.H. (2000). Motivation and learning strategies for college sucess. A selfmanagement approach. Mahwah, N.J.: LEA.

Dweck, C.S. (1986). Motivational processes affectong learning. American Psychologist, 41, 1040-1048.

Dweck, C.S. y Legget, E.L. (1988). A social-cognitive approach to motivation and personality. Psychological Review, 95, 256-273.

Eccles, J., Wigfield, A. y Schiefele, U. (1998). Motivation to succeed. En W. Damon y N. Eisenberg (Eds), Handbook of child psychology: Vol. 3, Social, Emotional and Personality Development (5 ed, pp. 1017-1095).

Elliot, A.J. (1997). Integrating the "classic" and "contemporary" approaches to achievement motivation: A hierarquical model of approach and avoidance achievement motivation. En Maerh y P.R. Pintrich (Eds), Advances in motivation and achievement (Vol. 10, pp. 143-179). Greenwich, CT: JAI Press.

Elliot, A. y Church, M. (1997). A hierarchical model of approach and avoidance achievement motivation. Journal of Personality and Social Psychology, 72, 218-232.

Elliot, A.J. y Harackiewicz, J.M. (1996). Approach and avoidance achievement goals and intrinsic motivation: A mediational analisys. Journal of Personality and Social Psychology, 70, 461-475.

Elliot, A.J., McGregor, H.A. y Gable, S. (1999). Achievement goals, study strategies, and exam performance: A mediational analysis. Journal of Educational Psychology, 91 (3), 549-563.

García, M.S., González-Pienda, J.A., Núñez, J.C., González-Pumariega, S., Alvárez, L., Roces, C., González-Cabanach, R. y Valle, A. (1998). El cuestionario de metas académicas (C.M.A.). Un instrumento para la evaluación de la orientación motivacional de los alumnos de Educación Secundaria. Aula Abierta, 71, 175-199. 
Gónzaléz-Pienda, J.A., Núñez, J.C., González-Pumariega, S., Álvarez, L., Roces, C., García, M., González, P. Cabanach, R.G. y Valle, A. (2000). Autoconcepto, proceso de atribución causal y metas académicas en niños con y sin dificultades de aprendizaje. Psichotema, 12 (4), 548-556.

Harackiewicz, J. M. y Barron, K. E. (1998). Rethinking achievement goals: when are they adaptive for college students and why?. Educational Psychologist, 33 (1), 1-21.

Harackiewicz, J.M., Barron, K.E. y Elliot, A.J. (1998). Rethinking achievement goals: When are they adaptative for college students and why?. Educational Psychologist, 33 (1), 121.

Harackiewicz, J.M., Barron, K.E., Carter, Letho y Elliot, A.J. (1997). Predictors and consequences of achievement goals in the college classroom: Maintaining interest and making the grade. Journal of Personality and Social Psychology, 73, 1284- 1295.

Harackiewicz, J.M., Barron, K.E., Tauer, J.M., Carter, S.M. y Elliot, A.J. (2000). Short-term and long-term consequences of achievement goals: Predicting interest and performance over time. Journal of Educational Psychology, 92 (2), 316-330.

Hidi, S. y Haracliewich, J.M. (2000). Motivating the academically unmotivated: A critical isue for the 21s century. Review of Educational Research, 70 (2), 151-179.

Kaplan, A. y Midgley, C. (1997). The effect of achievement goals: Does level of perceived academic-competence make a difference?.Contemporary Educational Psychology, 22 (4), 415-435.

Karabenick, S.A. y Collins-Eaglin, J. (1997). Relation of perceived instructional goals and incenyives to collegestudents' use of learning strategies. Journal of Experimental Educational, 65, 331-341.

Kuhl, J. (1984). Volitional aspects of achievement motivation and learned helplessness: Toward a comprehensive theory of action control. En B. Maher y W. Maher (eds.), Progress in experimental personality research (vol. 13, pp. 99-171). New York: Academic Press.

Kuhl, J. (1985).Volitional mediators of cognition-behavior consistency: Self-regulatory processess and action versus state orientation. En J. Kuhl y J. Beckman (eds.), Action control: From cognition behavior (pp. 101-128). New York: Springer-Verlag.

Kuhl, J. (1992). A theory of self-regulation: Action versus state orientation, selfdiscrimination, and some applications. Applied Psychology: An International Review, 41, 97-129. 
Maerh, M.L. y Meyer, H.A. (1997). Understanding motivation and schooling: Where we've been where we are, and where we need to go. Educational Psychology Review, 9, 399427.

Maehr, M. L., y Midgley, C. (1991). Enhancing student motivation: a schoolwide approach. Educational Psychologist, 26 (3 y 4), 399-427.

Maerh, M.L. y Midgley, C. (1996). Transforming school cultures. Boulder, CO: Westview.

Martin, A., Marsh, H. y Debus, R.L. (2001). Self-handicapping and defensive pessimism: exploring a model of predictors and outcomes from a self-protection perspective. Journal of Educational Psychology, 93 (1), 87-102.

Migdley, C. (1993). Motivation and middle level schools. En M.L. Maerh y P.R. Pintrich (eds.), Advances in motivation and achievement: vol. 8. Motivation in the adolescent years (pp. 217-274). Greenwich, CT: JAI.

Migdley, C., Arunkumar, R. y Urdan, T. (1996). “If I don't well tomorrow, there's a reason”: Predictors of adolescents' use of academic self-handicapping strategies. Journal of Educational Psychology, 88, 423-434.

Midgley, C., Kaplan, A., Middleton, M., Maehr, M.L., Urdan, T., Anderman, L.H., Anderman, E. y Roeser, r. (1998). The development and validation of scales assessing students' achievement goal orientations. Contemporary Educational Psychology, 23, 113-131.

Middleton, M. J., y Midgley, C. (1997). Avoiding the demonstration of lack of ability: an underexplored aspect of goal theory. Journal of Educatiional Psychology, 89 (4), 710 718.

Midgley, C. y Urdan, T. (1995). Predictors of middle school students' use of selfhandicapping strategies. Journal of Early Adolescence, 15, 389-411.

Midgley, C. y Urdan, T. (2001). Academic self-handicaping and achievement goals: A further examination. Contemporary Educational Psychology, 26 (1), 61-75.

Miller, A., Ferguson, E. y Byrne, I. (2000). Pupil's causal attributions for difficult classroom behaviour. British Journal of Educational Psychology, 70, 85-96.

Montero, I. y Alonso, J. (1992). Achievement motivation in high school: contrasting theoretical models in the classroom. Learning and Instruction, 2, 43-57.

Murphy, P.K. y Alexander, P.A. (2000). A motivated exploration of motivation terminology. Contemporary Educational Psychology, 25, 3-53.

Nicholls, J.G. (1984). Achievement motivation: Conceptions of ability, subjetive experience, task choice, and performance. Psychological Review, 91, 328-346. 
Newman, R.S. (1998). Students' help seeking during problem solving: Influences of personal and contextual achievement goals. Journal of Educational Psychology, 90 (4), 644658.

Núñez, J.C., González-Pienda, J.A., García, M.S., González-Pumariega, S., y García, S.I. (1995). Estrategias de aprendizaje en estudiantes de 10 a 14 años y su relación con los procesos de atribución causal, el autoconcepto y las metas de estudio. Revista Galega de psicopedagoxía, 10-11 (7), 219-242.

Paas, F., Camp. G. y Rikers, R. (2001). Instructional compensation for age-related cognitive declines: Effects of goals specificity in maze learning. Journal of Educational Psychology, 93 (1), 181-186.

Pajares, F. y Kranzler, J. (1995). Self-efficacy beliefs and general mental hability in mathematical problem-solving. Contemporary Educational Psychology, 20, 426-443.

Patrick, y Pintrich, (2001). Conceptual change in teachers' intuitive conceptions of learning, motivation, and instruction; the role of motivational and epistemological beliefs. En B. Torff y R.J. Sternberg, Understanding and teaching the intuitive mind (pp. 117-143). Mahwah, NJ: LEA.

Pintrich, P.R. (1999). Motivational beliefs as resources for and constraints on conceptual chage. En W. Schnotz, S. Vosniadou y M. Carretero, New Perspectives on Change Conceptual (pp.33-50). Amsterdan: Pergamon.

Pintrich, P.R. (2000a). The role of goal orientation in self-regulated learning. En M Boekaerts, P.R. Pintrich y M. Zeidner (eds.), The handbook of self-regulation (pp. 451-502). San Diego: Academic Press.

Pintrich, P.R. (2000b). Multiples Goals, Multiples Pathways: The role of Goal Orientation in learning and Achievement. Journal of Educational Psychology, 92 (3), 544-555.

Pintrich, P.R., y García, T. (1994). Regulating motivation and cognition in the classroom: The role of self-schemas and self-regulatory strategies. En D. Schunk y B. Zimmerman (Eds.), Self-regulation of learning and performance: Issues and educational applications. Hillsdale, NJ: Erlbaum.

Pintrich, P.R., y De Groot, E.V. (1990). Motivational and self-regulated learning components of classroom academic performance. Journal of Educational Psychology, 82, 33-40.

Pintrich, P.R. y Maerh, M.L. (Eds.) (1997). Advances in motivation and achievement, vol. 10. Greenwich, C.N.: JAI. 
Pintrich, P.R., y Schunk, D.H. (1996). Motivation in education: Theory, research, and applications (cap. 6: The role of goals and goal orientation). Upper Saddle River, NJ: Merrill/Prentice Hall.

Renzulli, J.S. y Yun, D. (2001). Abilities, interests, and styles as aptitudes for learning: A person-situation interaction perspective. En R.J. Sternberg y F.F. Zhang (eds.), Perspectives on thinking, learning, and cognitives styles (pp. 23-46). Mahwah, NJ: LEA.

Roces, C., González-Pienda, J.A., Núñez, J.C., González-Pumariega, S., García, S. y Álvarez, L. (1999). Relaciones entre motivación, estrategias de aprendizaje y rendimiento académico en estudiantes universitarios. Mente y Conducta en Situación Educativa, 1 (1), 41-50.

Roney,C., Higgins, E.T. y Shah, J. (1995). Goals and framing: how outcome focus influnces motivation and emotion. Personality Social Psychology Bulletin, 21, 1151-160.

Roney, C. y Sorrentino, R. (1995). Reducing self-discrepancies or maintaining selfcongruence? Uncertainty orientation, self-regulation, and performance. Journal of Personality Social Psychology, 68, 485-497.

Ryan, R.M. y Deci, E.L. (2000). Intrinsic and extrinsic motivations: classic definitions and new directions. Contemporary Educational Psychology, 25, 54-67.

Ryan, R.M. y Pintrich, P.R. (1997). “Should I as for help?”. The role of motivacion and attitudes in adolescents' help-seeking in math class. Journal of Educational Psychology, 89, 329-341.

Schunk, D.H. (1996). Goal and self-evaluative influences during children's cognitive skill learning. Am. Educational Research Journal, 33, 359-382.

Seifert, T. L. (1995). Characteristics of ego- and task-oriented students: a comparison of two methodologies. British Journal of Educational Psychology 65, 125-138.

Seifert, T.L. (1996). The stability of goal orientations in grade five students: Comparison of two methodologies. British Journal of Educational Psychology, 66 (1), 73-82.

Shah, J. y Kruglanski, A. (2000). Aspects of goals networks: Implications for self-regulation. En Boeckaerts, P.R. Pintrich y M. Zeider (Eds.), Handbook of self-regulation: Theory, research, and apllications (pp. 85-110). San Diego, C.A.: Academic Press.

Shneider, R.J., Ackerman,P.L. y Kanfer, R. (1996). To "act wisely in human relations": exploring the dimensions of social competence. Personality Individual Differences, 21, 469-481.

Skaalvik, E.M. (1993) Ego-involvement an self-protection among slow learniners: Four case studies. Scandinavian Journal of Educationa Research, 37, 305-315. 
Skaalvik, E.M. (1997). Self-enhancing and self-defeating ego orientation: Relations with task and avoidance orientation, achievement, self-perceptions, and anxiety. Journal of Educational Psychology, 89 (1), 71-81.

Slavin, R.E. (1983). When does cooperative learning increase student achievement?. Psychology Bulletin, 94, 429-445.

Smith, E.R. (1998). Mental representation and memory. En D. Gilbert, S. Fiske y G. Lindzey (eds.), The hanbook of social psychology ( $4^{\mathrm{a}}$ ed; vol 1, pp. 391-445). New York: oxford University Press.

Thompson, T. (1994). Self-worth protection: implications for the classroom. Educational Review, 46, 259-274.

Thompson, T., Davison, J.A. y Barber, J.G. (1995). Self-worth protection in achievement motivation: performance effects and attitudinal behaviour. Journal of Educational Psychology, 87, 598-610.

Thorkildsen, T.A. y Nicholls, J.G. (1998). Fifth graders' achievement orientations and beliefs: individual and classroom differences. Journal of Educational Psychology, 90 (2), 179201.

Urdan, T.C. (1997). Examining the relations among early adolescent students' goals and friends' orientation toward effort and achievement in school. Contemporary Educational Psychology, 22 (2), 165-191.

Urdan, T.C., Andeman, L. H., Anderman, E., y Roeser R. (1998). The development and Validation pf Scales Assessing Students' Achievement Goal Orientations. Contemporary Educational Psychology, 23, 113-131.

Urdan, T.C. y Maerh, M.L. (1995). Beyong a two-goal theory of motivation and achievement: A case for social goals. Review Educational Research, 65 (3), 213-243.

Urdan, T.C., Midgley, C., y Anderman, E.M. (1998). The role of classroom goal structure in students' use of self-handicapping strategies. American Educational Research Journal, 35, 101-122.

Valle, A., González, R., Gómez, M.L., Rodríguez. S. y Piñeiro, I. (1998). Influencia d elas atribuciones causales internas y externas sobre las metas académicas. Bordón, 50 (4), 405-413.

Volet, S. E. (1997). Cognitive and affective variables in academic learning: the significance of direction and effort in students' goals. Learning and Instruction, 7 (3), 235-254.

Walberg, H.J. (1981). A Psychology Theory of Educational Productivity. En Farney, F. y Gordon, N. (eds.), Psychology and Education. berckeley: McCutchan. 
Wentzel, K.R. (1991). Social and academic goals at school: Achievement motivation in context. En M. Maerh y Pintrich (Eds.), Advances in motivation and achievemenet (vol. 7, pp. 185-212). Greenwich, CT: JAI Press.

Wentzel, K.R. (1992). Motivation and achievemenet in adolescence: A multiple goals perspective. En D. Schunk y J. Meece (eds.), Students perception in the classroom: Causes and consecuences (pp. 287-306). Hillsdale, NJ: Erlbaum.

Wentzel, K.R. (1993).Social and academic goals at school: Motivation and achievement in early adolescence. Journal of Early Adolescence, 13, 4-20.

Wentzel, K.R. (1994). Relations of social goal pursuit to social acceptance, clasroom behavior, and perceived social support. Journal of Educational Psychology, 86, 173-182.

Wentzel, K.R. (1996). Motivation in context: Social relationships and achievemenet in middle school. En J. Juvonene y K.R. Wentzel (eds.), Social Motivation: Undestanding children's school adjustement (pp. 226-247). New York: Cambridge University Press.

Wentzel, K. R. (1997). Student motivation in middle school: the role of perceived pedagogical caring. Journal of Educational Psychology, 89 (3), 411-419.

Wentzel, K.R. (1998). Social relationships and motivation in middle school: The role of parents, teachers, and peers. Journal of Educational Psychology, 90 (2), 202-209.

Wentzel, K.R. (1999). Social-motivational processes and interpersonal relationships: implications for understanding motivation at school. Journal of Educational Psychology, 91 (1), 76-97.

Wentzel, K.R. (2000). What is it that I'm trying to achieve? Classroom goals from a content perspective. Contemporary Educational Psychology, 25, 105-115.

Wentzel, K.R. y Asher, S.R. (1995).Academic lives of neglected, rejected, popular, and controversial children. Child Development, 66, 754-763.

Wentzel, K.R. y Cadwell, K. (1997). Friendships, peer acceptance, and group membership: relations to academic achievement in middle school.Child Development, 68, 11981209.

Wentzel, K.R. y Wigfield, A. (1998). Academic and social motivational influences on students' academic performance. Educational Psychology Review, 10, 155-175.

Wigfield, A. y Eccles, J.S. (2000). Expectancy-Value Theory of Achievement Motivation. Contemporary Educational Psychology, 25, 68-81.

Wigfield, A., Eccles, J.S. y Pintrich, P.R. (1996)

Winne, P.H. (1997). Experimenting to bootstrap self-regulated learning. Journal of Educational Psychology, 89 (3), 397-410. 
Wolters, C.A. (1998). Self-regulated learning and college students' regulation of motivation. Journal of Educational Psychology, 90 (2), 224-235.

Zimmerman, B.J. (1994). Dimensions of academic self-regulation: A conceptual framewok for education. En D.H. Schunk y B.J. Zimmerman (Eds.), Self-regulation of learning and performance (pp. 154-180). Hillsdale, NJ: Erlbaum.

Zimmerman, B.J. (2000). Self-efficacy: an essential motive to learn. Contemporary Educational Psychology, 25, 82-91.

Zimmerman, B.J. y Bandura, A. (1994). Impact of self-regulatory influences on writing course attainment. American Educational Research Journal, 31, 845-862.

Zimmerman, B.J., Bandura, A. y Martínez-Pons, M. (1992). Self-motivation for academic attainment: The role of self-efficacy beliefs and personal golas setting. American Educational Research Journal, 29, 663-676.

Zimmerman, B.J. y Kintasas, A. (1997). Developmental phases in self-regulation: shifting from process goals to outcomes goals. Journal of Educational Psychology, 89 (1), 2936.

Zimmerman, B.J. y Kintasas, A. (1999). Acquiring writing revisison skill: Shifting from process to outcome self-regulatory goals. Journal of Educational Psychology, 91, 110.

Zimmerman, B.J. y Martínez-Pons, M. (1986). Development of a structures interview for assessing students use of self-regulated learning strategies. American Educational Research Journal, 23, 614-628.

Zimmerman, B.J. y Martínez-pons, M. (1990). Students differences in self-regulated learning: Relating grade, sex, and giftedness to self-efficacy and strategy use. Journal of Educational Psychology, 82, 51-59.

Zimmerman, B.J. y Risemberg, R. (1997). Self-regulatory dimensions of academic learning and motivation. En G.D. Phye (Ed.), Handbook of Academic Learning. Construction of Knowledge (pp.105-125). San Diego: Academic Press. 
Perspectivas recientes en el estudio de la motivación: la Teoría de la Orientación de Meta

[Página dejada en blanco intencionadamente] 